\title{
Dossier Interdisciplinarité Canevas pour une réflexion sur une interdisciplinarité entre sciences de la nature et sciences sociales
}

\author{
Marcel Jollivet ${ }^{\mathrm{a}}$, Jean-Marie Legay ${ }^{\mathrm{b}}$ \\ a Sociologue, LADYSS, Université de Paris X, bât. K, 92001 Nanterre cedex, France \\ b Biométricien, LBBE, Université Claude Bernard, Lyon 1, 43 boulevard du 11 novembre 1918, 69622 Villeurbanne cedex, France
}

Ces quelques mots d'ouverture n'ont d'autre objectif que de mieux cerner les contours des débats de ces journées ${ }^{1}$. Il ne s'agit bien sûr que de notre point de vue sur la façon d'aborder le thème qu'elles proposent à notre réflexion. Mais il peut être utile de se donner au moins un canevas de départ pour s'accorder sur les questions à aborder, quitte à le remettre, en fin de compte, en cause.

\section{Pour une science du dialogue entre les disciplines}

Une première précision s'impose concernant la terminologie. La notion d'interdisciplinarité est en effet utilisée dans de multiples sens et il est sans doute indispensable de s'accorder sur ce que nous entendrons ici par ce terme. Il s'agit bien pour nous d'une démarche de recherche construite en assemblant de façon méthodique des connaissances, des points de vue, des techniques de travail provenant de disciplines scientifiques différentes.

Trois points de cette définition méritent d'être soulignés et commentés : 1) il s'agit bien d'une "démarche", c'est-à-dire d'un processus de recherche à construire, et la grosse question est de savoir comment procéder à $l^{\prime}$ « assemblage » visé : l'expression «de façon méthodique " prend donc une particulière importance puisqu'elle renvoie directement à cette question et en souligne la dimension méthodologique; on pourrait dire

Auteur correspondant:

M. Jollivet, marcel.jollivet@u-paris10.fr

${ }^{1}$ Ce texte reprend les termes de l'exposé introductif fait par les auteurs à l'école thématique Ladyss/NSS-Dialogues «La démarche interdisciplinaire dans le domaine de l'environnement : méthodes et outils pour partager les savoirs » (Aussois, 29 septembre-2 octobre 2003) que toute recherche interdisciplinaire a un double objectif en termes de recherche : contribuer à la compréhension des phénomènes qui sont l'objet de la recherche, bien sûr, mais aussi faire progresser méthodologiquement la façon d'assembler, voire d'intégrer, les apports disciplinaires ; 2) ces apports consistent bien en « connaissances, points de vue, techniques de travail», et pas simplement en connaissances; la référence à la notion de "point de vue » est essentielle, elle inclut, au-delà des problématiques, ce qu'il est convenu d'appeler le «paradigme» des disciplines et également leurs principes épistémologiques, voire leur éthique ; 3 ) parler de démarche « de recherche ", c'est fixer un objectif d'explication au travail entrepris : là aussi se pose la question de savoir comment les apports des différentes disciplines sont combinés pour contribuer à cette explication; un rapport évident existe entre ce point et celui abordé en premier ; il va de soi qu'il ne saurait s'agir d'une simple juxtaposition de contributions disciplinaires, dans lequel cas on ne pourrait pas parler de « démarche d'assemblage méthodiquement construite » (il s'agirait de simple pluridisciplinarité, c'est-à-dire d'une juxtaposition de disciplines).

Ces trois prérequis, ou axiomes, renvoient à un principe commun, base même de cette interdisciplinarité, qui est le dialogue entre les disciplines : $c^{\prime}$ est à travers ce dialogue et seulement à travers lui que peut se construire une démarche méthodique, c'est-à-dire l'association ad hoc d'une problématique (et donc d'hypothèses) de recherche, d'une stratégie de recherche, d'objets de recherche et de modalités (y compris des techniques, mais pas seulement) d'interprétation interdisciplinaire. Parler de dialogue suggère un travail d'équipe associant des chercheurs de différentes spécialités. Il peut aussi s'agir d'un travail individuel, le chercheur se donnant 
les différentes cultures scientifiques qu'il souhaite associer. Mais les règles qu'il a à appliquer à son travail sont les mêmes que celles d'un collectif.

\section{Fausses querelles, vrais problèmes}

Contrairement à une idée répandue, cette démarche $\mathrm{d}^{\prime}$ assemblage interdisciplinaire n'est pas exceptionnelle. Une analyse fine des pratiques de recherche montrerait au contraire qu'elle est courante, pour ne pas dire banale. Mais, dans cet usage courant, les disciplines associées sont, dirons-nous, «proches » (encore que ce terme ne soit pas sans ambiguïté). Disons que leur complémentarité va tellement de soi, dans la logique même de la division du travail scientifique classique, que leur association, même novatrice, n'a nullement besoin de se qualifier d'interdisciplinaire et ne pose pas de problème épistémologique ou technique majeur. On pourrait parler à ce propos d'interdisciplinarité «de proximité ». C'est ce genre de pratique qui conduit parfois à la constitution de nouvelles disciplines (donc de disciplines hybrides, car cela existe). Il n'est donc pas sans intérêt ici de souligner que, dans la pratique, le travail scientifique n'a nullement le culte de la discipline, terme au demeurant d'un emploi souvent abusif et peu rigoureux : il la divise autant que de besoin en spécialités qu'il recombine en sautant même les frontières disciplinaires quand cela s'avère nécessaire pour faire avancer la recherche.

D'où vient alors le sentiment contraire, qui est à l'origine de ces journées, selon lequel l'interdisciplinarité est une pratique marginale, marginalisée par les institutions de recherche et dont la mise en œuvre est, de fait, difficile? Répondre à cette question conduit à préciser sous trois angles la nature des pratiques interdisciplinaires qui vont nous occuper. Il s'agit de la dynamique de recherche qui est à leur origine, des disciplines auxquelles elles font appel et enfin de leur rapport avec l'action.

\section{Une interdisciplinarité « exogène »}

En ce qui concerne le premier point, une distinction commode est souvent faite entre une recherche de type «fondamental » et une recherche procédant de la « demande sociale». Dans cette vision dichotomique des choses, l'interdisciplinarité serait une exigence créée par l'interférence entre demande sociale et science, le travail habituel de recherche étant au contraire caractérisé par une spécialisation croissante dans la discipline. De là à parler d'artefact à propos de cette interférence, il n'y a d'ailleurs qu'un pas vite franchi. Ce schéma n'est pas sans avoir quelque fondement si l'on se place sur un plan historiographique. On pourrait en effet retracer l'histoire d'une interdisciplinarité liée aux évolutions des politiques publiques : le cas de l'environnement est exemplaire, mais il n'est pas une exception. Mais, outre qu'elle ignore l'interdisciplinarité - de type fondamental - « de proximité » dont il vient d'être question, cette façon de voir considère à tort la recherche «fondamentale», y compris la plus spécialisée, comme une recherche totalement déconnectée de toute finalité sociale. L'interdisciplinarité n'est donc en soi ni une exception, on l'a déjà dit, ni un artefact, mais une méthode de recherche parfaitement intégrée à la pratique scientifique. Il reste que l'on peut dire que l'interdisciplinarité "de proximité » procède d'une dynamique endogène de la recherche, c'est-à-dire d'une sorte de «mûrissement » progressif et collectif des questions au sein de communautés scientifiques très spécialisées (on pourrait alors parler d'une «interdisciplinarité endogène »), alors qu'il existe en effet une interdisciplinarité résultant des changements dans les rapports entre science et société : une interdisciplinarité que l'on pourrait, en somme, qualifier $\mathrm{d}^{\prime}$ " exogène ».

En effet, que ce soit en raison de l'apparition de problèmes nouveaux à dimensions à la fois naturelles et sociales (et là encore la question de l'environnement est l'exemple par excellence, mais il n'est pas le seul) et/ou en raison d'une interaction à la fois plus rapide, plus forte, plus étendue et plus radicale dans ses effets, entre le développement des connaissances et les transformations de la société (pensons ici à la question des OGM ou à celle du clonage, par exemple), un besoin de connaissance nouveau apparaît à la jonction du scientifique et du sociétal. Ce besoin est bien directement lié au développement des connaissances scientifiques et de leurs applications, mais il ne découle pas directement de la logique du cheminement spontané classique du travail du chercheur. Au contraire, il en prend le contre-pied et le prend donc à contre-pied, quand il n'en apparaît pas comme une critique; en outre, il se manifeste comme une injonction venant du contexte social et, pire encore, de la sphère du politique et non pas de la communauté scientifique, seule autorité considérée par les chercheurs comme légitime pour définir les modalités de la production de la science. D'où la marginalité et la marginalisation institutionnelle de cette interdisciplinarité qui n'est pas, elle, « de proximité ».

Il convient bien évidemment de sortir le plus rapidement possible de ces oppositions manichéennes et superficielles, et d'y substituer un débat scientifique portant sur l'évaluation des différentes manières de faire de la recherche en les situant dans le mouvement de la science elle-même, dans l'évolution des sociétés et dans l'évolution de la place de la science dans celles-ci. Cette exigence doit d'autant plus être rappelée que cette interdisciplinarité qui n'est pas «de proximité » fait aussi partie des traditions académiques les plus classiques, dénuées de tout souci d'application (on pense en particulier à la géographie). Ce n'est pas le lieu de se lancer 
ici dans ce vaste chantier. Ce que nous pouvons simplement faire pour y contribuer durant ces journées, c'est de clarifier le plus possible les conditions de la mise en œuvre de cette interdisciplinarité, puisqu'aussi bien, ce qui découle clairement de ce bref examen, c'est qu'elle n'a pas été, elle, préparée par les habitudes de travail antérieures. On peut même dire que les cloisonnements que celles-ci ont instaurés contribueraient plutôt à accroître les difficultés auxquelles on se heurte pour la réaliser.

\section{Une interdisciplinarité « élargie »}

Cette tâche nous amène à aborder le deuxième registre des précisions nécessaires, car ces difficultés tiennent précisément à l'originalité des associations disciplinaires que cette interdisciplinarité requiert. Ceci découle de la dualité structurelle des problèmes qu'elle a à traiter. En effet, $\mathrm{du}$ fait qu'ils comportent intrinsèquement des dimensions naturelles et des dimensions sociales, il découle qu'elle doit combiner des disciplines traitant des unes et des autres. Il s'agit donc d'une interdisciplinarité qui n'a plus rien à voir avec une interdisciplinarité « de proximité ». Elle se complexifie en ayant vocation à associer toute la palette des disciplines, des sciences de la Terre aux sciences sociales en passant par les sciences de la vie. On pourrait la caractériser en parlant d'une interdisciplinarité « étendue » ou «élargie ». Elle a donc à mettre en rapport des phénomènes régis par des déterminismes de natures plus ou moins semblables ou différentes (physique, chimique, biologique, sociale); de ce fait, elle se heurte à toutes les difficultés que soulèvent les discontinuités cognitives que cette hétérogénéité et la division du travail de recherche qui s'est greffée dessus ont historiquement produites entre ces différents domaines de connaissance. Il est donc exclu qu'elle puisse faire l'impasse sur les problèmes d'ajustement entre disciplines qui en résultent.

L'exigence première à laquelle elle est confrontée et la tâche première qui lui incombe sont au contraire de mettre ces problèmes en évidence et de poser clairement la question de la façon de les traiter, c'est-à-dire soit d'en prendre tout simplement acte en les formulant clairement, soit de faire des propositions pour les résoudre, soit de les considérer comme irréductibles (au moins dans un terme prévisible). Là est la base même de sa rigueur. C'est en ce sens que toute recherche fondée sur une interdisciplinarité «étendue » a nécessairement une dimension méthodologique. Cette dimension devrait être explicitement affichée et faire l'objet d'un travail de recherche en soi. Pour lui donner corps, il est nécessaire de disposer d'une problématique portant sur les questions que soulèvent les rapprochements entre disciplines. On évoque souvent et depuis longtemps, mais sans jamais les approfondir vraiment, celle des incompatibilités des échelles d'espace et de temps ou celles résultant des décalages des vocabulaires. Ce sont en effet des questions importantes, mais il faudrait, à leur propos, aller au-delà d'un constat souvent sommaire et facilement négatif, qui est commodément utilisé pour prendre ses distances par rapport à l'interdisciplinarité, et apprendre au contraire à utiliser positivement les décalages d'analyse qui en résultent. Il faudrait en outre ne pas s'en tenir à elles, car elles ne sont pas les seules. Il existe à l'intérieur même des disciplines des «postures » de recherche différentes dont il devrait être tenu le plus grand compte, car elles sont plus ou moins favorables à l'ouverture et au dialogue entre disciplines. Des analyses portant sur des binômes de disciplines seraient instructives.

Reste au bout de toutes ces considérations une question fondamentale qui est la suivante : une construction interdisciplinaire «étendue » est-elle possible sans recours à une théorie ou à une méthodologie qui en fournisse les règles? Côté théorie, on pense évidemment à l'analyse systémique. Et, côté méthodologie, aux différentes formes de la modélisation. Ou bien est-il possible - et sage - d'en rester, au moins pour l'instant, à un bricolage aussi raisonné et éclairé que possible? Quel qu'il soit, un choix doit être fait; et ce choix doit être argumenté, car c'est toute la question de la généralisation, et donc de l'évaluation et de la validation de la démarche qui est en cause. Se contenter d'un pur pragmatisme du cas par cas la condamnerait sur le plan scientifique.

\section{Le rapport à l'action comme source de connaissance}

Le troisième angle sous lequel cette interdisciplinarité « étendue » doit être considérée, son rapport à l'action, renvoie à son origine la plus récente : dans la mesure où elle dérive d'une "demande sociale », c'est qu'il s'agit d'une question posée à la recherche en vue d'agir. C'est pourquoi on en parle comme d'une interdisciplinarité «finalisée », si ce n'est "appliquée ». Et il lui est, en effet, très souvent reproché - beau paradoxe! - de ne pas déboucher, justement, sur des conclusions utilisables pour l'action. Ce point de départ ouvre deux voies de recherche entre lesquelles le choix doit être clairement fait et annoncé. La première de ces voies consiste à chercher à aller le plus loin possible dans ce souci de répondre à une exigence d'action. L'idée de constituer les fondements d'une " recherche participative » représente l'exemple le plus achevé (même s'il n'a pas la nouveauté qu'il s'arroge souvent) de ce genre de préoccupation. Mais nombre d'autres voies sont explorées. Il s'agit bien là de recherche, car il est inévitable que les différentes formes d'association des "acteurs " à la recherche soulèvent des problèmes de recherche, tant quant à leur mise en œuvre pratique qu'en ce qui concerne la validation de leurs résultats (les deux points étant d'ailleurs liés). Bien évidemment, seules les opérations de recherche-action qui se fondent sur une interdisciplinarité «étendue » - et 
ce n'est pas nécessairement le cas de toutes - ont leur place dans notre réflexion autour de ces questions. La seconde voie consiste à considérer que le seul apport que puisse faire une recherche interdisciplinaire pour clarifier le rapport entre connaissance et action est de mener de front une analyse des dimensions naturelles et une analyse des dimensions sociales du problème à traiter et de montrer comment elles se conditionnent l'une l'autre, c'est-à-dire comment les dimensions naturelles et les dimensions sociales interagissent dynamiquement les unes sur les autres. On se situe là dans une perspective plus proche de la démarche de recherche "fondamentale » habituelle.

Ces deux voies ont un point commun : il est extrêmement souhaitable, pour ne pas dire indispensable, que les disciplines techniques compétentes fassent partie du «paquet» de disciplines associées. Ceci dit, elles doivent être nettement distinguées, car elles ne donnent pas la même place respective, ni le même rôle, aux acteurs et aux chercheurs dans le processus de recherche. La question des rapports que l'on pourrait éventuellement établir entre elles est posée : sont-elles ou ne sont-elles pas compatibles? Si elles le sont, ne pourraient-elles pas être utilisées de façon complémentaire, la seconde précédant - et éclairant-la première? Le fait que la seconde voie soit proche de la tradition universitaire ne doit pas occulter le fait que toutes constituent des voies de recherche à part entière, car elles posent toutes l'ensemble des questions que pose une interdisciplinarité «étendue ». Qui plus est, celles que l'on dit finalisées les posent en les polarisant, même si c'est de façon différente, sur les rapports entre connaissance et action, c'est-à-dire en traitant une autre question fondamentale et qui est généralement occultée derrière la notion de «transfert» de connaissances.

Cette dernière question est $\mathrm{d}$ 'autant plus vive et actuelle, mais aussi d'autant plus complexe, qu'elle implique désormais nécessairement une mise en perspective par rapport à la notion de développement durable. S'il est une notion qui contraint à penser le face-à-face entre les dimensions naturelles et sociales des problèmes de société en vue d'agir, c'est bien celle-ci. L'interdisciplinarité «étendue » est la seule voie de recherche qui permettre de prendre globalement en charge les attentes qu'elle induit, car ces attentes sont nécessairement au carrefour des dimensions écologiques et sociétales des problèmes. C'est aussi la seule voie de recherche qui puisse permettre de lui donner en fin de compte un contenu, c'est-à-dire de comprendre aussi bien les opportunités qu'elle ouvre que les contradictions qu'elle contient, et qui puisse mettre en évidence ses limites. Il s'agit, là encore, d'une facette intrinsèque - et originale - de cette interdisciplinarité «étendue ». Elle devrait, comme la facette méthodologique, être explicitée et donner lieu à des précisions concernant tant les protocoles de collecte des données que la nature de celles-ci et les orientations des analyses qui en sont faites, quant à la problématique de développement durable à laquelle ils renvoient. La première condition pour que les résultats $\mathrm{d}^{\prime} u n e$ recherche soient utilisables dans l'action n'est-elle pas que les données de base et les modalités de leurs traitements correspondent à la fois aux possibilités et aux contraintes auxquelles se trouvent confrontés les acteurs? Comment concilier cette nécessité avec celle de réaliser une analyse scientifique en soi, détachée de toute contingence autre que celle d'un travail de recherche de qualité? Toutes ces questions posées montrent que, à la fois indépendamment de son rapport à l'action et à travers lui, cette interdisciplinarité est bien fondamentalement une opération d'acquisition de connaissances. Finalisée ou pas, il ne faut pas oublier qu'il s'agit toujours d'une interdisciplinarité «cognitive » et que cette dimension doit être mise en évidence par les acquis qu'elle permet. Et ceci à la fois quant à la compréhension des faits étudiés, quant à la construction de règles de dialogue et de travail en commun entre les disciplines et, le cas échéant, quant à la place et à la nature de l'action à mener.

\section{Le cahier des charges du travail interdisciplinaire}

De tout ce qui précède, il ressort que prendre en charge dans sa globalité un problème qui comporte des dimensions naturelles et des dimensions sociales, et cela, à plus forte raison, dans une perspective d'action, c'est, pour un chercheur:

- accepter de s'interroger sur la place et le rôle de sa discipline dans un schéma d'analyse d'ensemble de ce problème ;

- s'intéresser à une réflexion méthodologique et théorique sur les rapports entre sa discipline et les autres disciplines mises à contribution pour le traiter ;

- contribuer à la conception, à l'expérimentation et à la validation de méthodes et de techniques destinées à intégrer les apports de sa discipline dans un schéma explicatif global;

- et avoir le souci d'inclure dans sa démarche de recherche - et donc de formaliser sur un plan théorique - les exigences de l'action conçue dans une perspective de développement durable.

Dans toutes ces tâches, il s'agit tout à la fois de chercher à tirer le bénéfice maximum des apports spécifiques et de maintenir les exigences de rigueur de sa discipline, mais aussi d'accepter d'en soumettre éventuellement les présupposés (le «paradigme») à la critique «externe», c'est-à-dire à la critique venant des autres disciplines, et d'explorer les voies d'innovations possibles facilitant le dialogue interdisciplinaire et enrichissant la discipline. La question immédiate, essentielle et difficile qui se pose au chercheur à travers la recherche de ces ajustements est celle, bien souvent mise en avant, là encore comme une 
limite de l'interdisciplinarité et une « excuse » justifiant de s'en garder, de savoir jusqu'où accepter le compromis avec les autres disciplines, voire un rôle de discipline ancillaire. La plupart du temps, cette attitude n'est que l'expression d'une paresse intellectuelle et du peu d'empressement à relever le défi que pose le jeu interdisciplinaire au confort de la posture disciplinaire : il n'est pas dans les habitudes ni considéré comme légitime qu'une discipline reprenne à son compte une question qui lui vient d'une autre. On ne saurait mieux illustrer la nécessité du dialogue dans l'exercice d'une interdisciplinarité «étendue », la place fondamentale qu'il occupe dans la construction de la démarche de recherche, de la mise au point de la problématique à l'interprétation des résultats, et le rôle qu'il a à jouer dans le retour sur les disciplines qu'entraîne la pratique interdisciplinaire. On ne saurait mieux montrer également qu'il s'agit bien d'une démarche d'ensemble faite d'étapes et de choix argumentés constituant une stratégie.
Ces tâches spécifiques du chercheur entrant dans une démarche d'interdisciplinarité étendue ne sont pas suffisamment objet d'attention et de réflexion. On tend en général à les passer sous silence, car on en reste au schéma selon lequel il n'y a que le "résultat », au sens factuel ou phénoménal du terme, qui compte. Mais la méthode compte aussi, surtout lorsqu'elle met en jeu des questions de fond, ce qui est en l'occurrence tout particulièrement le cas. La faire progresser est donc aussi un « résultat ». Une communauté scientifique existe lorsqu'elle partage une méthode. Et la question est tout particulièrement fondamentale pour l'interdisciplinarité qui nous réunit.

L'objectif majeur de ces journées ne devrait-il pas être de tirer des pratiques des uns et des autres, et de formuler clairement, quelques-unes des règles de cette méthode ou, au moins, les premiers principes sur lesquels fonder ces règles pour valider un savoir-faire et partager les questions qu'il pose afin de progresser collectivement?

To access this journal online: www.edpsciences.org 